NICETAE CHONIATAE HISTORIA

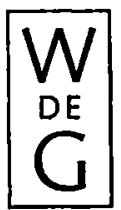




\section{CORPUS FONTIUM HISTORIAE BYZANTINAE}

CONSILIO SOCIETATIS INTERNATIONALIS STUDIIS BYZANTINIS PROVEHENDIS DESTINATAE EDITUM

VOLUMEN XI/1

\section{SERIES BEROLINENSIS}

EDIDERUNT

H.-G. BECK · A. KAMBYLIS · R. KEYDELL

APUD WALTER DE GRUYTER ET SOCIOS BEROLINI ET NOVI EBORACI MCMLXXV 


\title{
NICETAE CHONIATAE HISTORIA
}

\author{
RECENSUIT \\ IOANNES ALOYSIUS VAN DIETEN
}

PARS PRIOR

PRAEFATIONEM ET TEXTUM CONTINENS

APUD WALTER DE GRUYTER ET SOCIOS BEROLINI ET NOVI EBORACI MCMLXXV 
Gedruckt mit Unterstützung der Deutschen Forschungsgemeinschaft

CIP-Kurztitelaufnabme der Deutscben Bibliothek

Nicetas 〈Choniates〉

[Historia]

Nicetae Choniatae historia / rec. Ioannes

Aloysius van Dieten.

ISBN 3-11.004-528-1

P. 1. Praefationem et textum continens.

(Corpus fontium historiae Byzantinae;

Vol. 11, 1: Ser. Berolinensis)

(C)

1975 by Walter de Gruyter \& Co., vormals G. J. Göschen'sche Verlagshandlung - J. Guttentag,

Verlagsbuchhandlung - Georg Reimer - Karl J. Trübner - Veit \& Comp., Berlin 30

Printed in Germany

Alle Rechte, insbesondere das der Ubersetzung in fremde Sprachen, vorbehalten. Ohne ausdrüclliche Genehmigung des Verlages ist es auch nicht gestattet, dieses Buch oder Teile daraus auf photomechanischem Wege (Photokopie, Mikrokopie, Xerokopie) zu vervielfältigen.

Satz und Druck: Walter de Gruyter \& Co., Berlin 30

Buchbinder: Lüderitz \& Bauer, Berlin 